Теорія Ймовір. та Матем. Статист. Вип. 75, 2006
Theor. Probability and Math. Statist.

No. 75, 2007, Pages 179-188 S 0094-9000(08)00724-2

Article electronically published on January 25, 2008

\title{
ON STOCHASTIC STABILITY OF MARKOV DYNAMICAL SYSTEMS
}

UDC 519.21

\author{
JE. CARKOVS, I. VERNIGORA, AND V. YASINSKII
}

\begin{abstract}
This paper aims at discussing methods and results of Lyapunov stability theory for dynamical systems with vector field subjected to permanent Markov type perturbations. The paper is organized as follows. Section 1 introduces the model of Markov dynamical system (MDS) and suggests different possible definitions of equilibrium stochastic stability, which are under discussion in the next sections. It is proven that for linear Markov dynamical systems equilibrium asymptotical stability with probability one is equivalent to the exponential decreasing of the $p$-moment with sufficiently small $p$. In Section 3 we will discuss validity of equilibrium stability analysis of Markov dynamical systems applying a linear approximation of a vector field. Section 4 is devoted to a semigroup approach for mean square stability analysis of linear Markov dynamical systems. It permits us to write the Lyapunov matrix in an explicit form and to reduce the equilibrium stability problem to real spectrum analysis of a specially constructed closed operator.
\end{abstract}

\section{INTRODUCTION}

The dynamical system we shall deal with in this paper has the form of a quasilinear $n$-dimensional differential equation

$$
\frac{d x}{d t}=A(y(t)) x+f(x, y(t))
$$

where $y(t)$ is a homogeneous ergodic Markov process on metric compact phase space $\mathbb{Y}$ with weak infinitesimal operator $Q$ [2] and $f(x, y)$ is a continuous function with uniformly bounded $x$ derivative satisfying the condition $f(0, y) \equiv 0$. By the definition of Markov process, for any $s \geq 0$ and $y \in \mathbb{Y}$ there exists a unique stochastic process $y(t, s, y)$ satisfying the initial condition $y(s)=y$. Substituting any realization of this process in place of $y(t)$ into (1), we can analyze the resulting equation as an ordinary differential equation in $\mathbf{R}^{n}$ with dependent on $t$ (nonstationary) right hand side. It is not difficult to prove that for any above-mentioned realization and any $s \in \mathbf{R}$ and $x \in \mathbf{R}^{n}$ there exists a unique solution $x(t, s, x, y)$ of the Cauchy problem $x(s)=0$ for (1). The trivial solution $x(t) \equiv 0$ is the equilibrium point of (1), and we will discuss the behavior of other solutions starting in a sufficiently small vicinity of this equilibrium. We will say that equilibrium is

(1) locally almost surely stable if for any $s \in \mathbf{R}, \eta>0$, and $\beta>0$ there exists $\delta>0$ such that the inequality $\sup _{y \in \mathbb{Y}} \mathrm{P}\left(\sup _{t \geq s}|x(t, s, x, y)|>\eta\right)<\beta$ follows from the condition $x \in B_{\delta}(0)$, where $B_{\delta}(0):=\left\{x \in \mathbf{R}^{n}:|x|<\delta\right\}$;

2000 Mathematics Subject Classification. Primary 37H10, 34D20.

Key words and phrases. Markov dynamical systems, mean square stability, Lyapunov methods, limit theorems for random dynamical systems, stochastic differential equations.

(C)2008 American Mathematical Society 
(2) locally asymptotically stochastically stable if it is locally almost surely stable and there exists $\gamma>0$ such that the trajectories which do not leave the ball $B_{\gamma}$ tend to 0 as $t \rightarrow \infty$;

(3) asymptotically stochastically stable if it is locally almost surely stable and

$$
\lim _{T \rightarrow \infty} \sup _{y \in \mathbb{Y}} \mathrm{P}\left(\sup _{t>T}|x(t, s, x, y)|>c\right)=0
$$

for any $x \in \mathbf{R}^{n}, s \in \mathbf{R}$, and $c>0$;

(4) exponentially $p$-stable if there exist positive numbers $M$ and $\gamma$ such that

$$
\mathrm{E}|x(t, s, x, y)|^{p} \leq M|x|^{p} e^{-\gamma(t-s)}
$$

for any $x \in \mathbf{R}^{n}, y \in \mathbb{Y}, s \in \mathbf{R}$, and $t>s$.

We will call exponential 2-stability mean square stability.

\section{Stochastic Stability of Linear DiffEREnTial EQUATIOns WITH MARKOV COEFFICIENTS}

In this section we will deal with linear differential equations in $\mathbf{R}^{n}$

$$
\frac{d x}{d t}=A(y(t)) x
$$

where $A(y)$ is a continuous bounded matrix-valued function and $y(t)$ is a stochastically continuous Feller Markov process with weak infinitesimal operator $Q$. The pair $\{x(t), y(t)\}$ forms [10] a homogeneous stochastically continuous Markov process with the weak infinitesimal operator $L_{0}$ defined by the equality

$$
L_{0} v(x, y)=\left(A(y) x, \nabla_{x}\right) v(x, y)+Q v(x, y) .
$$

It is clear that there exists a family of the matrix-valued functions $\{X(t, s, y)\}$, defined by the equality $X(t, s, y) x=x(t, s, x, y)$, where $x(t, s, x, y)$ is the solution of the Cauchy problem $x(s, s, x, y)=x$ under the condition $y(s)=y$. The matrices $X(t, s, y)$ also satisfy equation (2) for all $t>s$ and initial condition $X(s, s, y)=I$, where $I$ is the matrix unit. This matrix family has the evolution property: $X(t, s, y)=X(t, \tau, y(\tau)) X(\tau, s, y)$ for any $y \in \mathbb{Y}, t \geq \tau \geq s \geq 0$. That allows us to define the Lyapunov $p$-index of (2) as

$$
\lambda^{(p)}=\sup _{x, y} \varlimsup_{t \rightarrow \infty} \frac{1}{p t} \ln \mathrm{E}|X(t, s, y) x|^{p} .
$$

It is not too difficult to prove that the exponential $p$-stability of the trivial solution of equation (2) is equivalent to the inequality $\lambda^{(p)}<0$. Because

$$
\left(\mathrm{E}|X(t, s, y) x|^{p_{1}}\right)^{1 / p_{1}} \leq\left(\mathrm{E}|X(t, s, y) x|^{p_{2}}\right)^{1 / p_{2}}
$$

for any positive $p_{1}<p_{2}$, the inequality $\lambda^{\left(p_{1}\right)} \leq \lambda^{\left(p_{2}\right)}$ follows from the inequality $p_{1}<p_{2}$ and therefore $\lambda^{(p)}$ is a monotone decreasing function of $p$. In this section we will prove that the asymptotic stochastic stability of (2) is equivalent to the condition

$$
\text { there exists } p_{0}>0 \text { such that } \lambda^{(p)}<0 \text { for all } p \in\left(0, p_{0}\right) .
$$

Lemma 1. If equation (2) is asymptotically stochastically stable, then it is exponentially p-stable for all sufficiently small positive $p$.

Proof. Let us put $\eta=1$ and $\beta=\frac{1}{2}$ in the definition of the almost sure stability and choose a positive $\alpha$ so small that the inequality

$$
\sup _{\substack{|x| \leq 2^{-\alpha} \\ y \in \mathbb{Y}}} \mathrm{P}\left(\sup _{t \geq 0}|X(t, 0, y) x|>1\right)<\frac{1}{2}
$$


is fulfilled. Due to the linearity of equation (2), one may deduce from the above inequality the inequality

$$
\sup _{|x| \leq 2^{-\alpha(l-1)}} \mathrm{P}\left(\sup _{t \geq 0}|X(t, 0, y) x|>2^{l \alpha}\right)<\frac{1}{2}
$$

for any $l \in \mathbb{N}, y \in \mathbb{Y}$. Let us denote

$$
g_{l}:=\sup _{|x| \leq 1, y \in \mathbb{Y}} \mathrm{P}\left(\sup _{t \geq 0}|X(t, 0, y) x| \geq 2^{l \alpha}\right) .
$$

The pair $\{x(t), y(t)\}$ is a stochastically continuous Markov process and one may apply the Markov property at the first moment $\tau_{1}(x)$ of exit of the trajectory $x(t, 0, x, y)$ from the ball $B_{1}(0)$ for any $x \in B_{1}(0)$. Hence

$$
\begin{aligned}
g_{l+1}= & \sup _{|x| \leq 1} \mathrm{P}\left(\sup _{t \in \mathbb{Y}}|X(t, 0, y) x| \geq 2^{(l+1) \alpha}\right) \\
\leq & \sup _{\substack{|x| \leq 2^{l \alpha} \\
y \in \mathbb{Y}}} \mathrm{P}\left(\sup _{t \geq 0}|X(t, 0, y) x|>2^{(l+1) \alpha}\right) \\
& \times \sup _{\substack{|x| \leq 1 \\
y \in \mathbb{Y}}} \iint_{s \geq 0,|u|=2^{l \alpha}} \mathrm{P}_{x \in \mathbb{Y}}\left(\tau_{1}(x) \in d s, x(s) \in d u, y(s) \in d v\right) \\
\leq & \frac{1}{2} \sup _{\substack{|x| \leq 1 \\
y \in \mathbb{Y}}} \mathrm{P}\left(\sup _{t \geq 0}|X(t, 0, y) x| \geq 2^{l \alpha}\right)=\frac{1}{2} g_{l} .
\end{aligned}
$$

Thus $g_{l} \leq 1 / 2^{l}$ for any $l \in \mathbb{N}$. Let us denote $\zeta:=\sup _{t \geq 0}|x(t, 0, x, y)|^{p}$. Because

$$
\begin{aligned}
\mathrm{E} \zeta & \leq|x|^{p} \sup _{|x| \leq 1} \mathrm{E} \zeta \leq \sum_{l=1}^{\infty} 2^{l \alpha p} \mathrm{P}\left(\sup _{t \geq 0}|x(t, 0, x, y)| \geq 2^{(l-1) \alpha}\right) \\
& \leq \sum_{l=1}^{\infty} 2^{l \alpha p} 2^{-l}|x|^{p}:=K_{1}|x|^{p},
\end{aligned}
$$

for all $p>0, x \in \mathbf{R}^{n}, y \in \mathbb{Y}$, one can determine that the random variable $\zeta$ has expectation for all $x \in \mathbf{R}^{n}, y \in \mathbb{Y}$, and $p \in\left(0, \alpha^{-1}\right)$. According to the conditions of the lemma, $x(t, 0, x, y)$ (as a solution of equation (2)) tends to 0 almost surely as $t \rightarrow \infty$ and uniformly on $y \in \mathbb{Y}$. Therefore, in view of the Lebesgue theorem,

$$
\lim _{t \rightarrow \infty} \sup _{y \in \mathbb{Y}} \mathrm{E}|x(t+s, s, x, y)|^{p}=0
$$

for all $x \in \mathbf{R}^{n}$ and $p \in\left(0, \alpha^{-1}\right)$. Besides, it is easy to verify that this convergence is uniform on $x$ within the ball $B_{1}(0)$ and for $s \geq 0$, i.e.,

$$
\lim _{t \rightarrow \infty} \sup _{x \in B_{1}(0), y \in \mathbb{Y}} \mathrm{E}|x(t+s, s, x, y)|^{p}=0 .
$$

Now we can choose a sufficiently large number $T$ in order that the inequality

$$
\sup _{y \in \mathbb{Y}} \mathrm{E}|x(t+s, s, x, y)|^{p} \leq|x|^{p} e^{-1}
$$

be fulfilled. Then because

$$
\begin{aligned}
\mathrm{E}|x(l T, 0, x, y)|^{p} & =\iint_{\mathbb{Y}, \mathbf{R}^{n}} \mathrm{P}(x, y,(l-1) T, d u, d v) \mathrm{E}|x(T, 0, u, v)|^{p} \\
& \leq e^{-1} \mathrm{E}|x((l-1) T, 0, x, y)|^{p},
\end{aligned}
$$


where $\mathrm{P}(x, y, t, d u, d v)$ is the transition probability of the homogeneous Markov process

$$
\{x(t), y(t)\},
$$

one can write

$$
\mathrm{E}|x(t, 0, x, y)|^{p} \leq K_{1} e^{-[t / T] T}|x|^{p},
$$

where $[a]$ is the integer part of a number $a$. This inequality completes the proof.

To analyze the behaviour of the solutions of equations (1) or (2), one may use the well-known Dynkin formula

$$
\mathrm{E}_{x, y}^{(u)} v\left(x\left(\tau_{r}(t)\right), y\left(\tau_{r}(t)\right)\right)=v(x, y)+\mathrm{E}_{x, y}^{(u)}\left\{\int_{u}^{\tau_{r}(t)}\left(L_{0} v\right)(x(s), y(s)) d s\right\},
$$

where the indices of expectation denote the condition $x(u)=x, y(u)=y$ and

$$
\tau_{r}(t)=\min \left\{\tau_{r}, t\right\}, \quad \tau_{r}=\inf \left\{t>u: x(t, u, x, y) \notin B_{r}(0)\right\} .
$$

Sometimes it is necessary to use the Lyapunov functions depending also on the argument $t$. If $v(t, x, y)$ as a function of the arguments $x$ and $y$ ) belongs to the domain of the infinitesimal operator $L_{0}$ and has continuous $t$ derivative, one may use the Dynkin formula (3) in the form

$\mathrm{E}_{x, y}^{(u)} v\left(\tau_{r}(t), x\left(\tau_{r}(t)\right), y\left(\tau_{r}(t)\right)\right)=v(u, x, y)+\mathrm{E}_{x, y}^{(u)}\left\{\int_{u}^{\tau_{r}(t)}\left(\frac{\partial}{\partial s}+L_{0}\right) v(s, x(s), y(s)) d s\right\}$.

To apply the second Lyapunov method, we will also use the well-known supermartingale inequality [1]

$$
\mathrm{P}\left(\sup _{t \geq u} \xi(t) \geq c\right) \leq \frac{1}{c} \mathrm{E} \xi(u)
$$

for the positive supermartingale $\{\xi(t)\}$.

Lemma 2. The trivial solution of equation (2) is exponentially p-stable if and only if there exists a Lyapunov function $v(x, y)$ satisfying the inequalities

$$
c_{1}|x|^{p} \leq v(x, y) \leq c_{2}|x|^{p}, \quad L_{0} v(x, y) \leq-c_{3}|x|^{p}
$$

for all $x \in \mathbf{R}^{n}, y \in \mathbb{Y}$ with some positive $p, c_{1}, c_{3}$.

Proof. Let there exist the above-mentioned Lyapunov function. It is easy to verify that

$$
\left(\frac{\partial}{\partial s}+L_{0}\right)\left(v(x, y) e^{\left(c_{3} / c_{2}\right) t}\right) \leq 0
$$

and then due to formula (4) one can write

$$
\mathrm{E}_{x, y} v(x(t), y(t)) e^{\left(c_{3} / c_{2}\right) t} \leq v(x, y) \leq c_{2}|x|^{p}
$$

for all $t, x$, and $y$. Hence

$$
\mathrm{E}_{x, y}|x(t)|^{p} \leq \frac{1}{c_{1}} e^{-\left(c_{3} / c_{2}\right) t} \mathrm{E}_{x, y} v(x(t), y(t)) e^{\left(c_{3} / c_{2}\right) t} \leq \frac{c_{2}}{c_{1}} e^{-\left(c_{3} / c_{2}\right) t}|x|^{p}
$$

and equation (2) is exponentially $p$-stable. Using the solutions $x(t+s, s, x, y)$ of equation (2), one can construct for any $T>0$ the function

$$
v(x, y):=\int_{0}^{T} \mathrm{E}|x(s+t, s, x, y)|^{p} d t
$$

which does not depend on $s$ owing to homogeneity of the Markov process $y(t)$. It is easy to verify that under the condition $\sup _{y \in \mathbb{Y}}\|A(y)\|:=a<\infty$ this function satisfies the 
first inequality of (4). If the trivial solution of equation (2) is exponentially $p$-stable, one can write the equality

$$
\begin{aligned}
L_{0} v(x, y) & =\lim _{\delta \rightarrow 0} \frac{1}{\delta}\left[\int_{0}^{T} \mathrm{E}_{x, y}\left\{\mathrm{E}_{x(\delta), y(\delta)}|x(t)|^{p}\right\} d t-\int_{0}^{T} \mathrm{E}_{x, y}|x(t)|^{p} d t\right] \\
& =\lim _{\delta \rightarrow 0} \frac{1}{\delta}\left[\int_{0}^{T} \mathrm{E}_{x, y}|x(t+\delta)|^{p} d t-\int_{0}^{T} \mathrm{E}_{x, y}|x(t)|^{p} d t\right]=\mathrm{E}_{x, y}|x(T)|^{p}-|x|^{p}
\end{aligned}
$$

and then

$$
L_{0} v(x, y) \leq\left(M e^{-\gamma T}-1\right)|x|^{p}
$$

where $M$ and $\gamma$ are constants from the definition of exponential $p$-stability. Now one can put $T=(\ln 2+\ln M) / \gamma$ and the proof is complete.

Corollary 1. If the trivial solution of (2) is exponentially p-stable, it is asymptotically stochastically stable.

Proof. Analyzing the proof of Lemma 2, one may conclude that the stochastic process $\zeta(t):=v(x(t), y(t)) e^{\left(c_{3} / c_{2}\right) t}$ is a positive supermartingale. Hence

$$
\begin{aligned}
\sup _{y \in \mathbb{Y}} \mathrm{P} & \left(\sup _{t \geq 0}|x(t, 0, x, y)|>\varepsilon\right)=\sup _{y \in \mathbb{Y}} \mathrm{P}\left(\sup _{t \geq 0}|x(t, 0, x, y)|^{p}>\varepsilon^{p}\right) \\
& \leq \sup _{y \in \mathbb{Y}} \mathrm{P}_{x, y}\left(\sup _{t \geq 0}\left\{\frac{1}{c_{1}} v(x(t), y(t))\right\}>\varepsilon^{p}\right) \\
& =\sup _{y \in \mathbb{Y}} \mathrm{P}_{x, y}\left(\sup _{t \geq 0}\left\{\frac{1}{c_{1}} \zeta(t) e^{-\left(c_{3} / c_{2}\right) t}\right\}>\varepsilon^{p}\right) \\
& \leq \sup _{y \in \mathbb{Y}} \mathrm{P}_{x, y}\left(\sup _{t \geq 0} \zeta(t)>\varepsilon^{p} c_{1}\right) \leq \frac{1}{\varepsilon^{p} c_{1}} \mathrm{E}_{x, y} \zeta(0) \leq \frac{c_{2}}{\varepsilon^{p} c_{1}}|x|^{p}
\end{aligned}
$$

and the trivial solution of (2) is stochastically stable almost surely. Now to prove asymptotic stochastic stability, one can apply the supermartingale inequality mentioned above and write the inequalities

$$
\begin{aligned}
\sup _{y \in \mathbb{Y}} \mathrm{P}\left(\sup _{t \geq u}|x(t, u, x, y)|>c\right) & \leq \sup _{y \in \mathbb{Y}} \mathrm{P}_{x, y}^{(u)}\left(\sup _{t \geq u}\left\{\frac{1}{c_{1}} v(x(t), y(t))\right\}>c^{p}\right) \\
& \leq \sup _{y \in \mathbb{Y}} \mathrm{P}_{x, y}^{(u)}\left(\sup _{t \geq u}\left\{\frac{1}{c_{1}} \zeta(t) e^{-\left(c_{3} / c_{2}\right) u}\right\}>c^{p}\right) \\
& \leq \frac{1}{c^{p} c_{1}} \mathrm{E} \zeta(u) \leq \frac{c_{2}}{c^{p} c_{1}}|x|^{p} e^{-\left(c_{3} / c_{2}\right) u} .
\end{aligned}
$$

\section{Stochastic Stability By Linear approximation}

In this section we will consider the quasilinear equation (1) under the conditions that the matrix $A(y)$ and the Markov process $y(t)$ satisfy the conditions of the previous section.

Theorem 1. If equation (2) is asymptotically stochastically stable and $\lim _{r \rightarrow 0} g_{r}=0$, then equation (1) is asymptotically stochastically stable.

Proof. Due to Lemma 1 and Lemma 2 we can construct the Lyapunov function $v(x, y)$ satisfying (4) with a small positive $p$. Since the matrix-valued function $D_{x} x(t, 0, x, y)$ is the Cauchy matrix of equation (2), it permits the estimation

$$
\sup _{y \in \mathbb{Y}} \mathrm{E}\left\|D_{x} x(t+s, s, x, y)\right\|^{p} \leq h_{2} e^{-\gamma t}
$$


with some positive constants $h$ and $\gamma$ for all $t>0$. Therefore the above Lyapunov function satisfies conditions (4) and, by construction, for all $x \neq 0$ has $x$ derivative satisfying the inequalities $\left|\nabla_{x} v(x, y)\right| \leq c_{3}|x|^{(p-1)}$ with some positive $c_{3}$. Because the above estimation does not depend on the initial time moment $s$, we will put for simplicity $s=0$. Denoting by $\{\tilde{x}(t)\}$ the solution of (1), one can estimate the function $L v(x, y)$ where $L$ is the weak infinitesimal operator of the pair $\{\tilde{x}(t), y(t)\}$ :

$$
\begin{aligned}
L v(x, y) & :=\left(A(y) x+g(x, y), \nabla_{x}\right) v(x, y)+Q v(x, y)=L_{0} v(x, y)+\left(g(x, y), \nabla_{x}\right) v(x, y) \\
& \leq-\frac{1}{2}|x|^{p}+c_{3}|x|^{p}|g(x, y)| \leq\left(g_{r} c_{3}-\frac{1}{2}\right)|x|^{p}
\end{aligned}
$$

for all $y \in \mathbb{Y}, x \in B_{r}(0)$, and $r>0$. Hence, due to the Dynkin formula, we may use the inequality

$$
\begin{aligned}
\mathrm{E}_{x, y}^{(u)} v\left(\tilde{x}\left(\tau_{r}(t)\right), y\left(\tau_{r}(t)\right)\right) & =v(x, y)+\mathrm{E}_{x, y}^{(u)}\left\{\int_{u}^{\tau_{r}(t)}(L v)(\tilde{x}(s), y(s)) d s\right\} \\
& \leq v(x, y)+\left(g_{r} c_{3}-\frac{1}{2}\right) \mathrm{E}_{x, y}^{(u)}\left\{\int_{u}^{\tau_{r}(t)}|\tilde{x}(s)|^{p} d s\right\}
\end{aligned}
$$

for all $y \in \mathbb{Y}, x \in B_{r}(0), r>0$, and $t \geq u \geq 0$. If $r$ is a sufficiently small number, the second summand on the right hand side of the last inequality is nonpositive. Hence the stochastic process $v\left(\tilde{x}\left(\tau_{r}(t)\right), y\left(\tau_{r}(t)\right)\right)$ is a supermartingale and we can write the inequalities

$$
\begin{aligned}
\mathrm{P}_{x, y}\left(\sup _{t \geq 0}|\tilde{x}(t)|>\varepsilon\right) & =\mathrm{P}_{x, y}\left(\sup _{t \geq 0}|\tilde{x}(t)|^{p}>\varepsilon^{p}\right)=\mathrm{P}_{x, y}\left(\sup _{t \geq 0}\left|\tilde{x}\left(\tau_{r}(t)\right)\right|^{p}>\varepsilon^{p}\right) \\
& \leq \mathrm{P}_{x, y}\left(\sup _{t \geq 0} v\left(\tilde{x}\left(\tau_{r}(t)\right), y\left(\tau_{r}(t)\right)\right)>c_{1} \varepsilon^{p}\right) \leq \frac{v(x, y)}{c_{1} \varepsilon^{p}} \leq \frac{c_{2} \delta^{p}}{c_{1} \varepsilon^{p}}
\end{aligned}
$$

for all $y \in \mathbb{Y}, x \in B_{\delta}(0), \delta \in(0, \varepsilon), \varepsilon \in(0, r)$, and sufficiently small $r>0$. The local almost sure stability immediately follows from these inequalities. Let us define the function

$$
h_{R}(r)= \begin{cases}1, & \text { for } x \in[0, R), \\ \frac{2 R-r}{R}, & \text { for } x \in[R, 2 R), \\ 0, & \text { for } x \geq 2 R\end{cases}
$$

The differential equation

$$
\frac{d x_{R}}{d t}=A(y(t)) x_{R}+h_{R}\left(\left|x_{R}(t)\right|\right) g\left(x_{R}, y(t)\right)
$$

has a unique solution of the Cauchy problem $x_{R}(0)=x$ because the function $h_{R}(|x|) g(x, y)$ satisfies the Lipschitz condition with constant $c_{2 R}$. Hence the pair $\left\{x_{R}(t), y(t)\right\}$ is a Markov process with the weak infinitesimal operator $L_{R}$ defined by the equality

$$
\begin{aligned}
L_{R} v(x, y) & =\left(A(y) x, \nabla_{x}\right) v(x, y)+\left(h_{R}(|x|) g(x, y), \nabla_{x}\right) v(x, y)+Q v(x, y) \\
& =L_{0} v(x, y)+\left(h_{R}(|x|) g(x, y), \nabla_{x}\right) v(x, y),
\end{aligned}
$$

and choosing $R$ small so that $\left(c_{2 R} c_{3}-\frac{1}{2}\right):=-c_{4}<0$, one can write the inequality $L_{R} v(x, y) \leq-c_{4}|x|^{p}$. Therefore

$$
\begin{aligned}
\mathrm{E}_{x, y}^{(u)} v\left(x_{R}(t), y(t)\right) & \leq v(x, y)-c_{4} \int_{u}^{t} \mathrm{E}_{x, y}^{(u)}\left|x_{R}(s)\right|^{p} d s \\
& \leq v(x, y)-\frac{c_{4}}{c_{1}} \int_{u}^{t} \mathrm{E}_{x, y}^{(u)} v\left(x_{R}(s), y(s)\right) d s
\end{aligned}
$$


for all $t \geq u \geq 0$. Hence the stochastic process $v\left(x_{R}(t), y(t)\right)$ is a positive supermartingale and one can write

$$
\begin{aligned}
\mathrm{P}_{x, y}\left(\sup _{t \geq s}\left|x_{R}(t)\right|>\varepsilon\right) & =\mathrm{P}_{x, y}\left(\sup _{t \geq s}\left|x_{R}(t)\right|^{p}>\varepsilon^{p}\right) \\
& \leq \mathrm{P}_{x, y}\left(\sup _{t \geq s} v\left(x_{R}(t), y(t)\right)>c_{1} \varepsilon^{p}\right) \\
& \leq \frac{1}{c_{1} \varepsilon^{p}} \mathrm{E}_{x, y} v\left(x_{R}(s), y(s)\right)
\end{aligned}
$$

for all $y \in \mathbb{Y}, x \in B_{R}(0), \varepsilon \in(0, R)$, and sufficiently small $R>0$. We can now get the inequality

$$
\mathrm{E}_{x, y} v\left(x_{R}(t), y(t)\right) \leq v(x, y) e^{-\left(c_{4} / c_{1}\right) t} \leq c_{2}|x|^{p} e^{-\left(c_{4} / c_{1}\right) t}
$$

from (5), and then in view of $\left(6^{\prime}\right)$ it can be written as

$$
\mathrm{P}_{x, y}\left(\sup _{t \geq s}\left|x_{R}(t)\right|>\varepsilon\right) \leq \frac{c_{2}|x|^{p}}{\varepsilon^{p} c_{1}} e^{-\left(c_{4} / c_{1}\right) s} .
$$

Hence all solutions of equation (6) which start at $t=0$ in the ball $B_{\varepsilon}(0)$ with $\varepsilon \in(0, R)$ and sufficiently small $R$ tend to 0 with probability one. Note that the solutions of equations (1) and (6) with the same initial conditions $x \in B_{\varepsilon}(0)$ coincide up to the moment when they leave the ball $B_{\varepsilon}(0)$. So any solution of (1) that does not leave the ball $B_{\varepsilon}(0)$ with sufficiently small $\varepsilon$ tends to zero with probability one, and the proof is complete.

\section{Mean square equilibrium stability of linear Markov dynamical systems}

In this section we will assume that $Q$ is a bounded operator or a closed operator of the class $\mathcal{H}(\gamma, 0)$ with some $\gamma>0$ (see [5]). Recall that $Q$ is a closed operator of the class $\mathcal{H}(\gamma, 0)$ if its resolvent exists inside the sector $S(\gamma, 0):=\left\{|\arg (\lambda)|>\gamma+\frac{\pi}{2}\right\}$ of the complex plane $\mathbf{C}$ and for any $\varepsilon>0$ and an interior point $\lambda \in S(\gamma-\varepsilon, 0)$ the inequality $\left\|(\lambda J-Q)^{-1}\right\| \leq M /|\lambda|$ is fulfilled for some positive $M$. Throughout this paper the operator $J$ is used to denote the unit operator. This section deals with the linear differential equations (2) under conditions of the previous section. We will study the conditions for equilibrium exponential mean square stability of this equation uniformly on $y \in \mathbb{Y}$, that is, the conditions for which the inequality $\mathrm{E}|x(t, s, x, y)|^{2} \leq M e^{-\rho(t-s)}|x|^{2}$ is fulfilled for some positive $M, \rho$ and any $t>s \geq 0, x \in \mathbf{R}^{n}, y \in \mathbb{Y}$. It will be proven that the latter is equivalent to the existence of a positive solution of a specially constructed Lyapunov equation for quadratic functionals on the space $\mathbf{R}^{n}$.

Let us denote by $\mathbf{M}$ the Banach space of symmetric $n \times n$ matrix-valued continuous functions $\{q(y), y \in \mathbb{Y}\}$ with norm defined by $\|q\|:=\sup _{y,|x|=1}|(q(y) x, x)|$. In this space one can define the set $\mathbf{K}:=\left\{q \in \mathbf{M}:(q(y) x, x) \geq 0\right.$, for all $x \in \mathbf{R}^{n}$, for all $\left.y \in \mathbb{Y}\right\}$ of nonnegative definite matrices. For two elements $q_{1}$ and $q_{2}$ of the space $\mathbf{Q}$ we shall write $q_{1} \gg q_{2}$ if $q_{1}-q_{2} \in \mathbf{K}$. If for some positive $c$ one can write $(q(y) x, x) \geq c|x|^{2}$ for all $x \in \mathbf{R}^{n}$ and $y \in \mathbb{Y}$, then $q$ is called an inner point of $\mathbf{K}$. The set of all inner points of $\mathbf{K}$ will be denoted $\tilde{\mathbf{K}}$. Due to the homogeneity of the process $\{x(t), y(t)\}$ the Cauchy matrices $X(t+u, s+u, y)$ and $X(t, s, y)$ have the same probability characteristics for any $u \geq 0, t \geq s \geq 0$, and $y \in \mathbb{Y}$. Hence, one can introduce a one-parameter family of operators $\{T(t), t \geq 0\}$ in the space $\mathbf{M}$ by the equality

$$
(T(t) q)(y)=\mathrm{E}_{y}^{(s)}\left\{X^{T}(t+s, s, y) q(y(t+s)) X(t+s, s, y)\right\} .
$$

Lemma 3. The family of operators $\{T(t), t \geq 0\}$ forms a strongly continuous semigroup [3] with infinitesimal operator defined by $(\mathbf{A} q)(y)=A^{T}(y) q(y)+q(y) A(y)+Q q(y)$. 
Proof. The semigroup property follows from the equalities

$$
\begin{aligned}
(T(t+u) q)(y) & \\
& =\mathrm{E}_{y}\left\{\mathrm{E}_{y(u)}^{(u)}\left\{X^{T}(u, 0, y) X^{T}(t+u, u, y(u)) q(y(t+u)) X(t+u, u, y(u)) X(u, 0, y)\right\}\right\} \\
& =(T(u)(T(t) q))(y) .
\end{aligned}
$$

The infinitesimal operator of the semigroup $\{T(t)\}$ is defined [3] by the equality

$$
\begin{aligned}
(\mathbf{A} q)(y) & =\lim _{t \rightarrow 0}\left[\mathrm{E}_{y}\left\{X^{T}(t, 0, y) q(y(t)) X(t, 0, y)\right\}-q(y)\right] / t \\
& =A^{T}(y) q(y)+q(y) A(y)+Q q(y) .
\end{aligned}
$$

The operator $\mathbf{A}$ is the sum of the operator $Q \in \mathcal{H}(\gamma, 0)$ and the bounded operator $A^{T}(y) q(y)+q(y) A(y)$. Hence [5], $\mathbf{A}-\beta J \in \mathcal{H}\left(\gamma_{1}, 0\right)$ (or, using the notation of [5], $\left.\mathbf{A} \in H\left(\gamma_{1}, \beta\right)\right)$ with some $\gamma_{1}$ and $\beta$, and the semigroup generated by this operator is strongly continuous.

Lemma 4. If the spectrum $\sigma(\mathbf{A})$ of the operator $\mathbf{A}$ is situated in the half-plane $\mathbf{C}_{\alpha}:=$ $\{\mathbf{C}: \operatorname{Re} \lambda \leq \alpha\}$ and there exists an eigenvalue with real part equal to $\alpha$, then $\alpha$ also is an eigenvalue.

Proof. Under the previous assumptions the operator A forms a continuous semigroup $T(t)$ described [5] by the equality $T(t)=(2 \pi i)^{-1} \int_{\Gamma} e^{\lambda t}(J \lambda-\mathbf{A})^{-1} d \lambda$, where the integral on the right hand side is defined along some contour $\Gamma$. Due to this representation we can write the formula $P \sigma(T(t))=e^{t P \sigma(\mathbf{A})}$, where $P \sigma$ denotes the point part of the spectrum. The resolvent $(J \lambda-\mathbf{A})^{-1}$ also can be represented as

$$
(J \lambda-\mathbf{A})^{-1}=\int_{0}^{\infty} e^{-\lambda t} T(t) d t
$$

for all $\lambda \notin \mathbf{C}_{\alpha}$. If $\varphi$ is an eigenvalue of the operator $\mathbf{A}$ with $\operatorname{Re} \varphi=\alpha$ and $q$ is the corresponding eigenfunction, then

$$
(J \lambda-\mathbf{A})^{-1} q=(\lambda-\varphi)^{-1} q .
$$

Let us assume that $\alpha \notin P \sigma(\mathbf{A})$. For all real $\lambda>\alpha,|x|=1$, and $q \in \mathbf{K}$ we can write the inequality

$$
0 \leq \int_{0}^{\infty} e^{-\lambda t}(T(t) q(y) x, x) d t \leq \lim _{\mu \rightarrow \alpha} \int_{0}^{\infty} e^{-\mu t}(T(t) q(y) x, x) d t \leq\left\|(J \alpha-\mathbf{A})^{-1}\right\| \cdot\|q\| .
$$

It can be clearly seen that any real uniformly bounded on $y \in \mathbb{Y}$ symmetric matrix $q(y)$ can be represent as the difference of the positive matrix $q_{1}(y) \equiv\|q\| I$ and the nonnegative matrix $q_{2}(y)=q_{1}(y)-q(y)$. Hence, for any $q \in \mathbf{M}$ there exist $q_{1}, q_{2} \in \mathbf{K}$ such that $q=q_{1}-q_{2}$ and $\left\|q_{1}\right\|+\left\|q_{2}\right\| \leq 3\|q\|$. By the definition of continuous operator $C_{0}$-semigroup [3] we may use the inequality

$$
\begin{aligned}
\left|e^{-\lambda t}(T(t) q(y) x, x)\right| & \leq e^{-\operatorname{Re} \lambda t}\left(T(t) q_{1}(y) x, x\right)+e^{-\operatorname{Re} \lambda t}\left(T(t) q_{2}(y) x, x\right) \\
& \leq 3\|q\| e^{-\alpha t}(T(t) I x, x)
\end{aligned}
$$

for all complex $\lambda \notin \mathbf{C}_{\alpha}$ and $q \in \mathbf{M}$ and therefore, one can conclude that the limit $\lim _{\lambda \rightarrow \varphi}(J \lambda-\mathbf{A})^{-1}$ exists as $\lambda$ tends to $\varphi$ from the half-plane $\{\operatorname{Re} \lambda>\alpha\}$. Because one can reach the same conclusion for a complex-valued symmetric continuous matrix $q(y)$, this result leads to a contradiction.

Theorem 2. The trivial solution of equation (2) is exponentially mean square stable if and only if there exist $q \in \tilde{\mathbf{K}}$ and $r \in \tilde{\mathbf{K}}$ such that $\mathbf{A} q=-r$. 
Proof. If the trivial solution of equation (2) is exponentially mean square stable, there exists the quadratic form of $x$ defined by the equality

$$
v(x, y):=\int_{0}^{\infty} \mathrm{E}_{y}|X(t, 0, y) x|^{2} d t:=\int_{0}^{\infty}((T(t) I)(y) x, x) d t:=(q(y) x, x),
$$

where $q \in \mathbf{M}$. Let $a=\sup _{y \in \mathbb{Y}}\|A(y)\|$. Then, the inequality

$$
\left|\frac{d \mathrm{E}|x(t, 0, x, y)|^{2}}{d t}\right| \leq 2 a \mathrm{E}|x(t, 0, x, y)|^{2}
$$

may be written for any $t \geq 0, y \in \mathbb{Y}, x \in \mathbf{R}^{n}$ and one can conclude that there exist positive numbers $c_{1}$ and $c_{2}$ such that $c_{1}|x|^{2} \leq(q(y) x, x) \leq c_{2}|x|^{2}$ for any $x \in \mathbf{R}^{n}, y \in \mathbb{Y}$, that is, $q:=\{q(y), y \in \mathbb{Y}\} \in \tilde{\mathbf{K}}$. Besides, by construction $r:=-\mathbf{A} q=I \in \tilde{\mathbf{K}}$. Let us now assume that there exist $q$ and $r$ as mentioned in the theorem. Because the pair $\{x(t), y(t)\}$ is a homogeneous right continuous Markov process with weak infinitesimal operator $L$, by the definition of the function $v(x, y)=(q(y) x, x)$ one can write the equality

$$
L v(x, y)=\left(\left[A^{T}(y) q(y)+q(y) A(y)+Q q(y)\right] x, x\right)=((\mathbf{A} q)(y) x, x)=-(r(y) x, x) .
$$

For the above function $v(x, y)$ one can use the Dynkin formula in the form

$$
\mathrm{E}_{y}(q(y(t)) x(t), x(t))=(q(y) x, x)-\int_{0}^{t} \mathrm{E}_{y}(r(y(s)) x(s), x(s)) d s .
$$

Since $q \in \tilde{\mathbf{K}}$ and $r \in \tilde{\mathbf{K}}$, there exist positive numbers $c_{1}, c_{2}$, and $c_{3}$ such that $c_{1}|x|^{2} \leq$ $(q(y) x, x) \leq c_{2}|x|^{2},(r(y) x, x) \geq c_{3}|x|^{2}$ for any $x \in \mathbf{R}^{n}, y \in \mathbb{Y}$. Therefore, one can deduce the inequality

$$
\begin{aligned}
\mathrm{E}_{y}(q(y(t)) x(t), x(t)) & =(T(t) q(y) x, x)=(q(y) x, x)+\int_{0}^{t}(T(s)(\mathbf{A} q)(y) x, x) d s \\
& \leq c_{2}|x|^{2}-\int_{0}^{t}(T(s) r(y) x, x) d s \leq c_{2}|x|^{2}-c_{3} \int_{0}^{t} \mathrm{E}_{y}|x(s)|^{2} d s
\end{aligned}
$$

or

$$
c_{1} \mathrm{E}_{y}|x(t)|^{2} \leq c_{2}|x|^{2}-c_{3} \int_{0}^{t} \mathrm{E}_{y}|x(s)|^{2} d s .
$$

Then for any $x \in \mathbf{R}^{n}, y \in \mathbb{Y}$,

$$
\mathrm{E}_{y}|x(t)|^{2} \leq \frac{c_{2}}{c_{1}}|x|^{2} e^{-\left(c_{3} / c_{1}\right) t}
$$

and the proof is completed.

Let us define the potential $R$ (see [2]) of the semigroup $T(t)$ by the equality

$$
(R q)(y):=\int_{0}^{\infty} T(t) q(y) d t
$$

We will write $q \in D(R)$ if the above integral converges according to the norm of the space $\mathbf{M}$.

Corollary 2. Equation (2) is exponentially mean square stable if and only if $I \in D(R)$.

Proof. Let us assume that $I \in D(R)$. Due to the inequality

$$
\left|e^{\lambda t}(T(t) q(y) x, x)\right| \leq 3\|q\|(T(t) I x, x) e^{\operatorname{Re} \lambda t}
$$

the potential is a bounded linear operator and $\sigma(\mathbf{A}) \subset\{\mathbf{C}: \operatorname{Re} \lambda \leq 0\}$. Hence [3], A $\in \mathcal{H}(\gamma,-\rho)$ and $\|T(t)\| \leq M e^{-\rho t}$ with some positive $\rho$ and $M$, and inequality (8) follows from the inequality

$$
\mathrm{E}_{y}|x(t)|^{2}=\mathrm{E}\left(X^{T}(t, 0, y) X(t, 0, y) x, x\right)=((T(t) I)(y) x, x) \leq\|T(t) I\| \cdot|x|^{2} .
$$


Next, let the trivial solution of equation (2) be exponentially mean square stable. Then, due to the inequality

$$
(T(t) I x, x)=\mathrm{E}\left(X^{T}(t, 0, y) X(t, 0, y) x, x\right)=\mathrm{E}_{y}|x(t)|^{2} \leq M|x|^{2} e^{-\rho t}
$$

one can write that $I \in D(R)$. Thus, the proof is completed.

The equation

$$
A^{T}(y) q(y)+q(y) A(y)+Q q(y)=-I
$$

is called the Lyapunov equation for mean square stability investigation of equation (2).

\section{BIBLIOGRAPHY}

1. J. L. Doob, Stochastic Processes, John Willey \& Sons, New York, 1953. MR0058896 (15:445b)

2. E. Dynkin, Markov Processes, Academic Press, New York, 1965. MR0193671 (33:1887)

3. E. Hille and R. Philips, Functional Analysis and Semigroups, AMS Colloquium Publications, vol. 34, AMS, Providence, 1957. MR0089373 (19:664d)

4. L. Katafygiotis and Ye. Tsarkov, Mean square stability of linear dynamical systems with small Markov perturbations. I. Bounded coefficients, Random Oper. and Stoch. Equ. 2 (1996), no. 4, 149-170. MR 1399076 (98h:60084)

5. T. Kato, Perturbation Theory for Linear Operators, Springer-Verlag, Berlin-Heidelberg, 1966. MR $0203473(34: 3324)$

6. R. Has'minskii, Stochastic Stability of Differential Equations, Kluwer, Norwell, MA, 1980. MR600653 (82b:60064)

7. V. S. Korolyuk and A. V. Swishchuk, Semi-Markov Random Evolution, Mathematics and its Applications, vol. 308, Kluwer Academic Publishers, Dordrecht, 1995. MR.1472977(98e:60145)

8. M. G. Krein and M. A. Rutman, Linear operators leaving invariant a cone in a Banach space, Uspehi Matem. Nauk (N.S.) 3 (1948), no. 3, 3-95. (Russian) MR0027128(10:256e)

9. V. I. Oseledec, A multiplicative ergodic theorem. Lyapunov characteristic numbers for dynamical systems, Transactions of Moscow Mathematical Society 19 (1968), 197-231. MR0240280 (39:1629)

10. A. V. Skorokhod, Asymptotic Methods of the Theory of Stochastic Differential Equations, AMS, Providence, RI, 1989. MR,1020057 (90i:60038)

11. K. Sadurski and Ye. Tsarkov, On diffusion approximation and stochastic stability, Theory Stoch. Process. 2(18) (1996), no. 1-2, 81-95.

12. Ye. F. Tsarkov, Averaging and stability of cocycles under dynamical systems with rapid Markov switching, Exploring Stochastic Laws (A. V. Skorokhod and Yu. V. Borovskih, eds.), VSP, Utrecht, The Netherlands, 1995, pp. 469-479. MR1714028(2000i:37076)

Department of Theory Probability and Mathematical Statistics, Riga Technical UniverSity, Meza Street, 1/4, Riga, LV-1048, Latvia

E-mail address: carkovs@livas.lv

Department of Computer Sciences, Chernivtsi National University, Kotsyubyns'kil Street, 2, Chernivtsi, 58000, Ukraine

E-mail address: irchik78@ukr.net

Department of Computer Sciences, Chernivtsi National University, Kotsyubyns'kĭ Street, 2, Chernivtsi, 58000, Ukraine

E-mail address: yasik@ukrtel.net

Received 24/NOV/2004

Originally published in English 